\title{
Prakash Kafle
}

MS, MCh Neurosurgery

Department of Neurosurgery,

Nobel Medical College\& Teaching Hospital,

Kathmandu Nepal

\section{Sushil Krishna Shilpakar}

MS Neurosurgery

Professor of Neurosurgery,

HOD, Department of Neurosurgery,

Institute of Medicine, TU Teaching Hospital,

Kathmandu Nepal

Mohan Raj Sharma

MS Neurosurgery

Professor of Neurosurgery

Department of Neurosurgery

Institute of Medicine, TU Teaching Hospital,

Kathmandu Nepal

\section{Gopal Sedain}

MS, MCh Neurosurgery

Assistant Professor of Neurosurgery

Department of Neurosurgery,

Institute of Medicine, TU Teaching Hospital,

Kathmandu Nepal

\section{Amit K Pradhanang}

MS MCh Neurosurgery

Assistant Professor of Neurosurgery

Department of Neurosurgery

Institute of Medicine, TU Teaching Hospital,

Kathmandu Nepal

Binod Raj bhandari

MBBS, MS

MCh Neurosurgery Resident

Department of Neurosurgery, TUTH

\section{Address for correspondence:}

Dr.Gopal Sedain

MS MCh Neurosurgery

Assistant Professor of Neurosurgery

IOM, TUTH, Kathmandu Nepal

Email: neuron79@gmail.com

Date received: $16 / 3 / 18$

Date accepted: $27 / 4 / 18$

$\mathrm{J}$

oubert syndrome is a rare autosomal recessive genetic neurodevelopmental disorderthat affects the area of brain that controls balance and coordination. ${ }^{6}$ The common typical clinical manifestations are abnormal respiratory pattern (hyper apnea), occulomotor findings, low muscle tone (hypotonia), lack of muscle control (ataxia), developmental retardation with evidence of neuropathologic abnormalities of cerebellum and

\section{Joubert Syndrome: A Case Report}

Joubert syndrome (JS) isa rare autosomal recessiveneurodevelopmentaldisorderinvolving cerebellarvermis and brainstem,marked byagenesis of cerebellar vermis, ataxia, hypotonia,oculomotor apraxia, neonatal breathingproblems and mental retardation. Magnetic Resonance Imaging (MRI) revealsthe characteristic Molar tooth sign of midbrain and Batwing appearance of rostral fourthventricle.

Key Words: Joubertsyndrome, hypotonia, vermianagenesis, molar tooth sign, bat wing appearance 


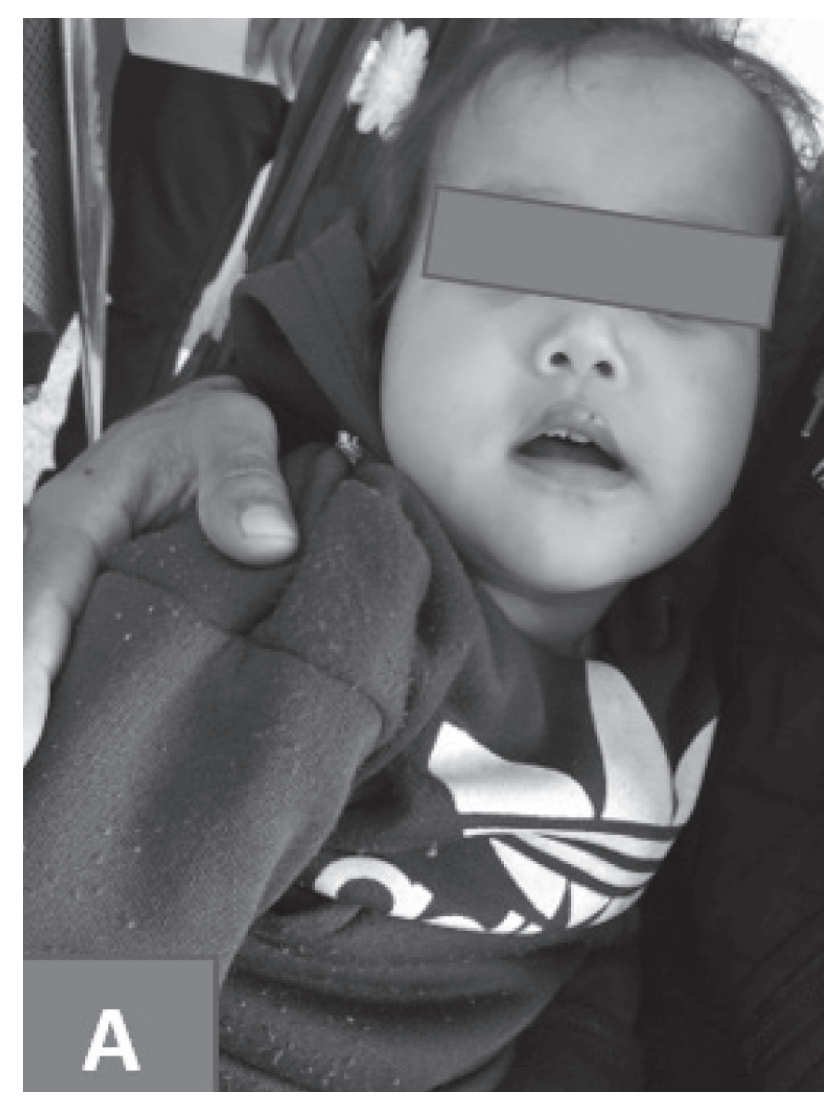

Figure 1.A: Baby with Joubert Syndrome

findings which are now known to be caused by defects in the structure and/or function of the primary cilium. ${ }^{12}$

The incidence of JS has been estimated to be between $1 / 80,000$ and $1 / 100,000$ live births. ${ }^{7}$ It is a condition with a variable phenotype making it difficult to establish the full clinical diagnostic spectrum of symptoms of the syndrome.

Even though the clinical features of the disorder are present in the newborn period, the correct diagnosis is often not made for several months or years after birth. ${ }^{1}$ The average age at diagnosis is 33 months. ${ }^{10}$ The importance of early detection of the syndrome is emphasized; so that suitable measures can be started as early as possible. Many children with JS exhibit dysmorphic facial features that include broad forehead, arched eyebrows, eyelid ptosis, wide-spaced eyes, open- mouth configurationand facial hypotonia. ${ }^{11}$

The clinical course can be variable; but most children with this disorder survive infancy to reach adulthood. We report a case of Joubert syndrome who presented with delayed developmental milestones, abnormal body posture, hypotonia and typically demonstrating diagnostic Molar toothsign (MTS) and Batwing appearance on Magnetic resonance imaging(MRI)in which the

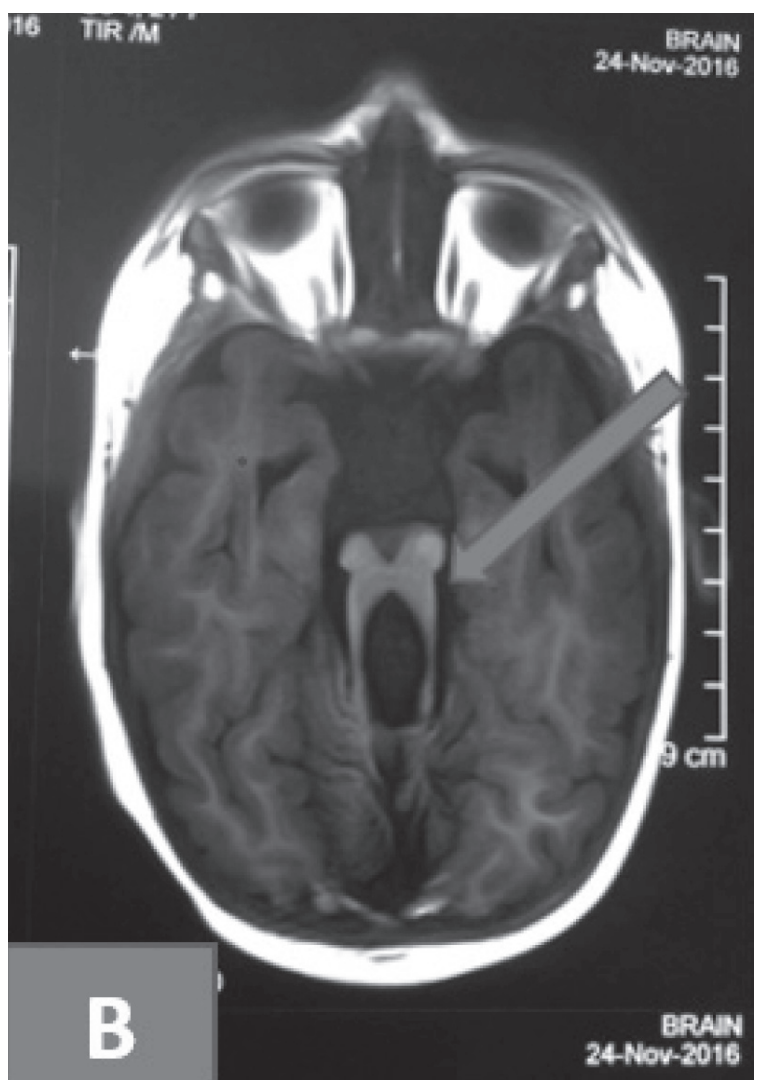

Figure 1.B:The Molar tooth sign appearance of Ponto cephalic junction. Arrow head.

cerebellar vermis of the brain is underdeveloped and the brain stem is abnormal.

\section{Case Detail}

A 3-year-old male child was brought to the NeurosurgicalOut-Patient Clinic by his parents with chief complaints of delayed developmental milestones and growth retardation, difficulty in holding his head and abnormal movements. The boy was only able to sit, walk and speak at 15, 28 and 48 monthsrespectively which indicatedsignificant delay indevelopmentalmilestone. There was no abnormal antenatal history. The child was delivered in a local hospital at term vianormal vaginal route. There was no significant perinatal period. He had seizure attacks soon after birthand has been under anticonvulsant medication ever since. His parentshad no history of consanguinous marriage.

The boy was restless and abnormally moving his head to and fro. On physical examination, hewashypotonic; but had no dysmorphic facial features. Head circumference was within normal range forage. There were no abnormalities detected on cardiovascular and respiratory system examinations.Similarly,gastrointestinal and genitourinary 
system revealed normal findings.His picture is as shown in (Figure 1.A).

Routine hematology, urinalysis, echocardiography and thyroid function testswereall unremarkable.

MRI scan of brain revealed small brainstem with volume loss involving the left half of brainstemand hypoplasticcerebellar vermis. The Molar tooth sign appearance of pontocephalicjunction (Figure 1.B) and Batwing appearance of the rostral fourth ventricle were observed on the scan.

The genetic analysis was not performed however, the parents have been advisedfor further metabolic and chromosomal study on follow up. At present, the child ison regular follow up in Neurosurgical Out-Patient Clinic.

\section{Discussion:}

Joubert syndrome is inherited as a rare autosomal recessive disorder, characterized by a specific congenital malformation of the hindbrain and a broad spectrum of other phenotypic findings. ${ }^{12}$ Its association with supratentorial anomalies are uncommon; but cerebral cortical dysplasia and gray matter heterotopias have been reported in the literature. ${ }^{3}$ Moderate lateral ventricular enlargement due to atrophy has been described in 6-20\% of cases. ${ }^{9}$ Many authors have reported the prevalence of some of these associated findings, which include extra fingers and toes - polydactyly (8\%), abnormality of iris - ocular coloboma (4\%), and hamartomas of the tongue $(2 \%)$, dysmorphicfacies, microcephaly, tongue protrusion, polycystic kidney disease, congenital heart disease, unsegmented midbrain tectum, retinal dystrophy and agenesis of the corpus callosum. ${ }^{5}$ In our case, all these aforementioned associated features were absent, suggestive it to be pure JS.

Though diagnosis of JS is generally based on presence of typical clinical features and the "molar tooth sign" as seen on an MRI, the definitive diagnostic criteria for JS isyet to beestablished. However, the clinical features frequently considered as essential for the diagnosis of classic JS comprise the following: ${ }^{8}$

1. Hypotonia in infancy.

2. Delayed developmental milestone/mental retardation.

3. One or both of the following:

a. Irregular breathing pattern in infancy.

b. Abnormal eye movements.

In our case, all of the above features were present except for irregular breathing pattern.

Joubert Syndromeand Related Disease(JSRD) are classified into six phenotypic subgroups: pure JS;
JS with ocular defect; JS with renal defect; JS with oculorenaldefects; JS with hepatic defect and JS with orofaciodigital defects. ${ }^{2}$ Our case was consistent with the pure JS phenotypic subgroup.

Important radiological findings of JS include deep interpeduncular fossa, narrow isthmus (the pontomesencephalic junction), lack of decussation of superior cerebellar peduncles, dilated, distorted, and rostrally deviated fourth ventricle giving the typical "Batwing" appearance, thick vertical superior cerebellar peduncles, rostral deviation of fastigium of fourth ventricle, wide foramen of Magendie and dysplastic vermis. The brainstem, predominantly the medulla and upper cervical spinal cord, tends to be small. "Molar tooth sign" encompasses deeper than normal posterior interpeduncular fossa, prominent or thickened superior cerebellar peduncles, and vermian hypoplasia or dysplasia. ${ }^{9}$

Genetically, the causative genes have been identified, all encoding for proteins of the primary cilium or the centrosome, making JSRD part of an expanding group of diseases called (Ciliopathies). ${ }^{4}$ Mutations in the AHI1 gene are the cause in $10-15 \%$ of the cases. Mutation in the CEP 290 (NPHP6) gene is in10\%. Homozygous deletion of NPHP1 gene is in 1-2\%. Fetalultrasonography may be useful. Fetal MRI is the diagnostic method of choice. ${ }^{2}$ Developmental outcome in JS is variable. Steinlin, et $a l,{ }^{13}$ suggested that outcomes in JS can be categorized into three courses: first, children who die young; second, patients who survive but are severely developmentally delayed and have a variety of visual and motor handicaps; and third, patients whose developmental quotients fall within the mildly delayed range (70-80). Taking into consideration of the neurological deficits, our case belongs to the third subgroup with mildly delayed milestone.

In children with JSRD, managing respiratory and feeding problems related to breathing difficultiesandhypotoniamust be given utmost priority. Prolonged apneacan be life-threatening. Sometimes, the respiratory abnormalities may resolve spontaneously. Prognosis depends basically on the severity of breathing dysfunction, renal and hepatic complications. After the first months of life, overall prognosis varies considerably among JSRD subgroups, depending on the extent and severity of organ involvement. ${ }^{4}$

Generally, the treatment strategy for JS is symptomatic and supportive.Developmental delays and retardation are usually managed with proper physical therapy, occupational therapy, speech therapy and infant stimulation.Children with JS should be evaluated by numerous specialists on multidisciplinary basis, including nephrologists, ophthalmologists, geneticists and neurologists. Routine 


\section{Kafle et al}

screening is recommended for liver, kidney and retinal abnormalities.Genetic counselling should be recommended for those with JS and their families.

\section{Conclusion:}

Joubert syndrome is arare autosomal recessive neurodevelopmental disorder.Consanguineous marriage carries increased risk. MRI of the brain is diagnostic, demonstrating the characteristic molar tooth appearance of midbrain and bat wing configuration of the rostral fourth ventricle. Patients with JS presents with episodes of abnormal respiratory pattern, oculomotor findings, hypotonia, ataxia, developmental retardation with evidence of neuropathologic abnormalities of cerebellum and brainstem.Diagnosis is based on typical clinicoradiological features. Managing respiratory and feeding problems related to breathing abnormalities or hypotoniashould be given the utmost priority. Overall treatment is basically symptomatic and supportive.

\section{References:}

1. Akcakus M, Gunes T, Kumandas S, Kurtoglu S, Coskun A. Joubert syndrome: Report of a neonatal case. Paediatr Child Health 8:499-502,2003

2. Adamsbaum C, Moutard ML, André C, Merzoug V, Ferey S, Quéré MP, Lewin F, Fallet-Bianco C. MRI of the fetal posterior fossa. Pediatr Radiol 35:12440,2005

3. Barkovich AJ. Pediatric neuroimaging 2nd ed. New York, NY: Raven; pp. 249-57, 1995
4. BrancatiF,DallapiccolaB,Valente EM. Joubert Syndrome and related disorders. Orphanet J Rare Dis 5:20, 2010

5. Chance PF, Cavalier L, Satran D, Pellegrino JE, Koenig M, Dobyns WB. Clinical nosologic and genetic aspects of Joubert and related syndromes. J Child Neurol 14:660-6, 1999

6. Edwin JR. van Beek, Charles BLM. Majoie. Case 25: Joubert Syndrome. Radiology 216:379-82, 2000

7. Harjinder Gill, BrindaMuthusamy, DenizeAtan, Cathy Williams and Matthew Ellis. Joubert Syndrome Presenting with Motor Delay and Oculomotor Apraxia. Case Reports in Pediatrics 2011

8. Joubert M, Eisenring JJ, Robb JP, Andermann F. Familial agenesis of the cerebellar vermis. Asyndrome of episodic hyperpnea, abnormal eye movements, ataxia and retardation. Neurology 19:813-25, 1969

9. Kendall B, Kingsley D, Lambert SR, Taylor D, Finn P. Joubert syndrome: A clinico-radiological study. Neuroradiology 31:502-6, 1990

10. Maria BL, Quisling RG, Rosainz LC, Yachnis AT, Gitten J, Dede D, et al. Molar tooth sign in Joubert syndrome: Clinical, radiologic, and pathologic significance. J Child Neurol 14:368-76, 1999

11. Melissa A. Parisi. Clinical and molecular features of Joubert syndrome and related disorders. Am J Med Genet C Semin Med Genet 151: 326-340,2009

12. Parisi MA. Clinical and molecular features of Joubert syndrome and related disorders.Am J Med Genet C Semin Med Genet 151:326-40, 2009

13. Steinlin M, Schmid M, Landau K, Boltshauser E. Follow-up in children with Joubert syndrome. Neuropediatrics 28:204-11, 1997 